\title{
Aplicaciones clínicas de la teoría integral de la continencia
}

\author{
C. Riccetto, P. Palma, A. Tarazona \\ Universidade Estadual de Campinas, UNICAMP, Campinas, São Paulo. Brasil. \\ Universidad El Bosque. Colombia. \\ Actas Urol Esp 29 2005; (1): 31-40
}

\section{RESUMEN}

APLICACIONES CLÍNICAS DE LA TEORÍA INTEGRAL DE LA CONTINENCIA

En la actualidad, el tratamiento de la incontinencia de orina no se puede realizar sin tener en cuenta la teoría integral de la continencia. Esta teoría considera que la incontinencia a los esfuerzos, la urgencia y las alteraciones del vaciamiento vesical se presentan por las alteraciones sucedidas en los elementos de soporte suburetral, de los ligamentos y de los músculos del suelo pélvico. Las alteraciones de la tensión aplicada por los músculos y ligamentos sobre las fascias yuxtapuestas a la pared vaginal determinan la apertura o el cierre del cuello vesical y de la uretra. Alteraciones de la tensión sobre la vagina determinan, también, la activación prematura del reflejo miccional, desencadenando contracciones involuntarias del detrusor.

El conocimiento de los fundamentos anatómicos del piso superior, intermedio e inferior del suelo pélvico así como de los principales elementos ligamentosos pubouretrales, uretropélvicos y úterosacros son esenciales antes de abordar cualquier cirugía de la incontinencia.

Dependiendo de la localización de la lesión músculo fascial o ligamentar y de la sensibilidad de las terminaciones nerviosas locales, se puede desarrollar incontinencia de esfuerzo, incontinencia de urgencia, alteraciones del vaciamiento vesical o varias combinaciones de estas condiciones. Existen 6 defectos básicos que deben ser investigados sistemáticamente: defecto del soporte suburetral (“Hammock"), sindrome de vagina fija ("Tethered vagina syndrome”), distensión de los ligamentos pubouretrales, distensión de los ligamentos útero sacros y del soporte del ápice vaginal, lesiones de la inserción vaginal de los músculos pubococcígeos, lesiones de los músculos estriados del piso pélvico por traumatismo del esfinter externo del ano o distensión, parálisis o ruptura de las inserciones del plató elevador.

Palabras clave: Anatomía suelo pélvico, Incontinencia urinaria de esfuerzo, Teoría integral continencia.

\section{ABSTRACT \\ CLINICAL APPLICATIONS OF THE COMPREHENSIVE THEORY OF URINARY INCONTINENCE}

Currently, urinary incontinence cannot be treated without taking into consideration the comprehensive theory of urinary incontinence. According to this theory, stress incontinence, urge incontinence and alterations in bladder emptying result from alterations in the elements of suburethral support, ligaments and muscles of the pelvis floor. Alterations in the forces applied by muscles and ligaments to the fascia adjacent to the vaginal wall and the urethra cause the vesical neck and the urethra to open or close. Alterations in the forces applied to the vagina can also cause premature activation of the micturition reflex, triggering involuntary contractions of the detrusor muscle.

Knowledge of the anatomic substrate of the upper, intermediate and lower layers of the pelvic floor and of the main pubourethral, urethropelvic and uterosacral ligamentous elements is essential before any surgical approach of incontinence can be attempted.

Depending on the location of the fascial or ligamental muscle lesion and of the sensitivity of the local nerve endings, stress incontinence, urge incontinence, altered bladder emptying or combinations of these conditions can develop. There are 6 basic defects which should be systematically investigated: defect in the suburethral support ("Hammock"), tethered vagina syndrome, distended pubourethral ligaments, distended uterosacral ligaments and support of the vaginal apex, lesions of vaginal insertion of pubococcygeal muscles, lesions of the striated muscles of the pelvic floor levator plate.

Key words: Pelvic floor anatomy. Urge urinary incontinence. Comprehensive theory of incontinence. 
$\mathrm{L}$ a Teoría Integral de la Continencia fue concebida por Peter Petros y Ulmsten, con el objetivo de explicar de forma integral los mecanismos fisiopatológicos involucrados, no solamente en la incontinencia urinaria de esfuerzo, sino de los sintomas habitualmente coexistentes, tales como la urgencia, polaquiuria, nocturia, alteraciones del vaciamiento vesical e intestinal, además del dolor pélvico crónico. Esa teoría considera que dichos sintomas son interdependientes, dentro de un proceso fisiopatológico común.

La Teoría Integral de la Continencia considera básicamente que la incontinencia a los esfuerzos, la urgencia y las alteraciones del vaciamiento vesical se presentan por las alteraciones sucedidas en los elementos de soporte suburetral, de los ligamentos y de los músculos del suelo pélvico. De acuerdo con esa Teoría, las alteraciones de la tensión aplicada por los músculos y ligamentos sobre las fascias yuxtapuestas a la pared vaginal determinan la apertura o el cierre del cuello vesical y de la uretra. Alteraciones de la tensión sobre la vagina determinan, también, la activación prematura del reflejo miccional, desencadenando contracciones involuntarias del Detrusor.

Basada en la interpretación conjunta de los conocimientos anatómicos y funcionales obtenidos por diversos autores a lo largo del siglo pasado, la Teoría Integral propone una nueva clasificación para la incontinencia urinaria de la mujer, fundamentada en tres zonas de disfunción y seis defectos principales, que deben ser sistemáticamente explorados y simultáneamente corregidos durante el tratamiento quirúrgico. De esta forma, las disfunciones miccionales resultantes del tratamiento quirúrgico de la incontinencia urinaria resultarían del abordaje incompleto de dichos defectos.

\section{FUNDAMENTOS ANATÓMICOS}

Componentes musculares y de la fascia

Los elementos músculo-fasciales del perineo femenino actúan de forma conjunta. Entretanto, tres músculos y tres ligamentos sirven de base para el funcionamiento ideal de los mecanismos de micción, evacuación y de continencia.

Clásicamente, los músculos del piso pélvico femenino son clasificados anatómicamente en dos grupos: el diafragma urogenital, mas externo, tenue y restringido a la región anterior del periné, y el diafragma pélvico, mas robusto y localizado mas internamente. Basado en estudios funcionales al respecto de la dinámica de los órganos pélvicos, la musculatura del piso pélvico puede, además, ser clasificada en 3 componentes básicos (Fig. 1):

a) Camada superior: Con la contracción en dirección horizontal, con participación en el mecanismo de continencia. Está representada fundamentalmente por el músculo pubococcigeo (Contracción en dirección anterior) y por el plató del músculo elevador del ano (Contracción en dirección posterior) (Fig. 2).

b) Camada intermedia: Con contracción en sentido caudal (para abajo), responsable de las angulaciones del recto, la vagina y del cuerpo vesical. El principal componente de esta camada es el músculo longitudinal externo del ano. (Fig. 3).

c) Camada inferior: Con contracción horizontal, tiene función únicamente de soporte de los componentes más externos del aparato genital femenino, representado por el diafragma urogenital.

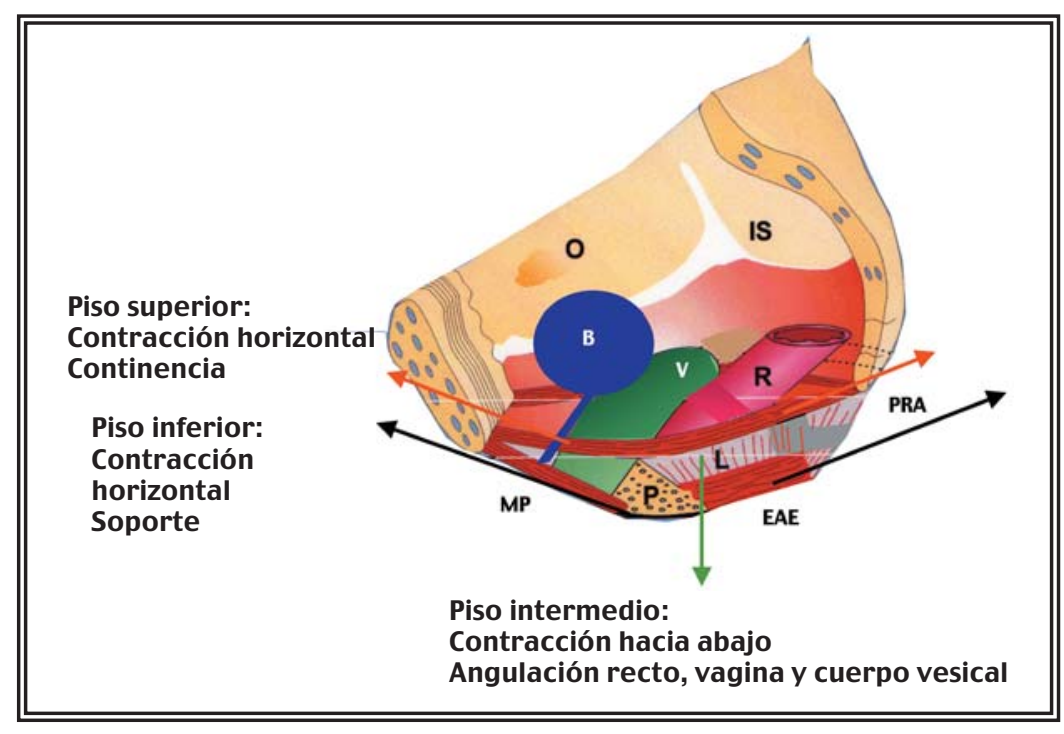

FIGURA 1. Clasificación funcional de las camadas musculares del piso pélvico en la mujer. PM: membrana perineal; PRA: plató retro-anal; L: músculo longitudinal del ano; EAE: esfinter estriado del ano; V: vagina; P: cuerpo perineal; R: recto; IS: isquio; O: obturador. 


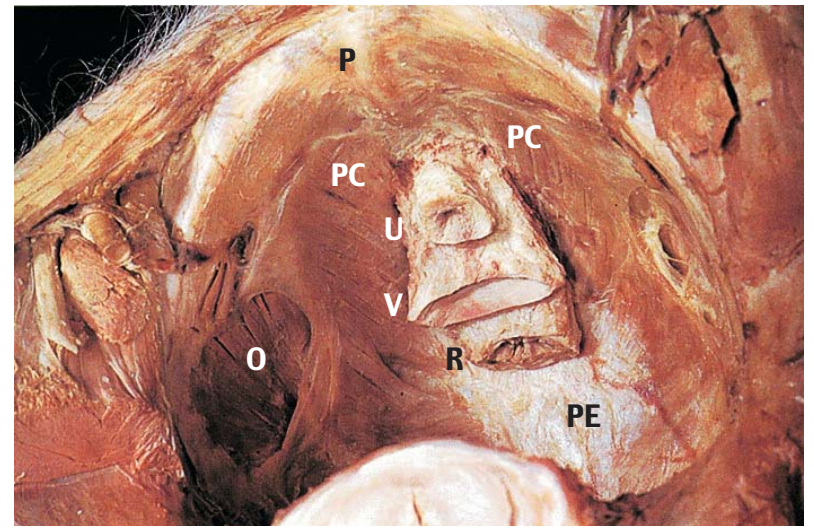

FIGURA 2. Cara superior del diafragma pélvico. P: pubis, U: uretra; V: vagina; R: recto; PC: eje pubococcigeo del músculo elevador del ano; PE: plató del músculo elevador del ano; O: músculo obturador.

\section{Principales ligamentos}

Los elementos de soporte ligamentar del piso pélvico femenino interactúan con los músculos, presentando una función dinámica, conferida tanto por su elasticidad como por los mecanismos de micción, evacuación y de continencia.

Se destacan tres ligamentos principales (Fig. 4):

a) Ligamentos pubouretrales: tienen su origen en el borde inferior del pubis, presentando porciones, prepúbica y retropúbica. Insertándose bilateralmente en el arco tendíneo de la fascia pélvica, y a nivel del tercio medio de la uretra.

b) Ligamentos uretropélvicos: de composición fibro-muscular, sus extremos se originan bilateralmente en los ligamentos pubouretrales, en su punto de inserción en la fascia pubocervical, y se funden en la región central, constituyendo el principal elemento de soporte suburetral. Actúan en conjunto con los ligamentos pubouretrales en los mecanismos de continencia y de micción.

c) Ligamentos útero sacros: $\mathrm{Se}$ originan bilateralmente en la cara anterior del sacro y se insertan en la fascia pubocervical en el ápice vaginal, integrando el anillo pericervical.

La región comprendida entre el tercio de los ligamentos pubouretrales (tercio uretral medio) y el cuello

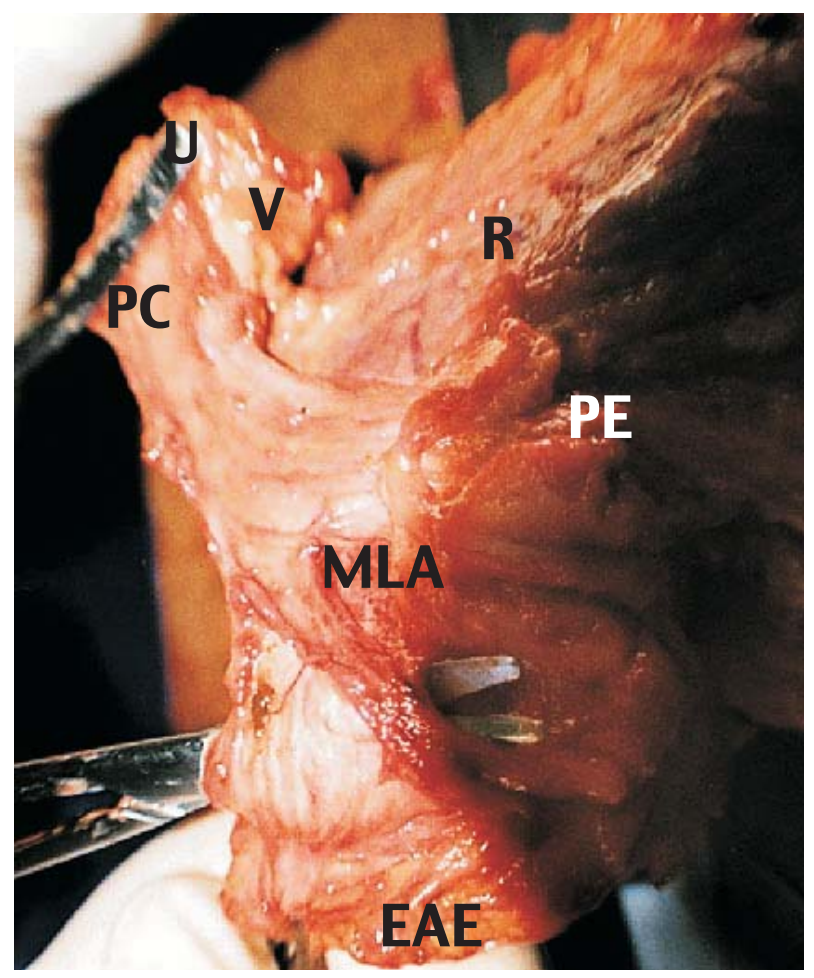

FIGURA 3. Camada muscular intermediaria del piso pélvico femenino. U: uretra; V: vagina; $R$ : recto; PC: eje pubococcígeo del músculo elevador del ano; PE: plató del músculo elevador del ano; MLA: músculo longitudinal del ano; EAE: esfinter anal externo.

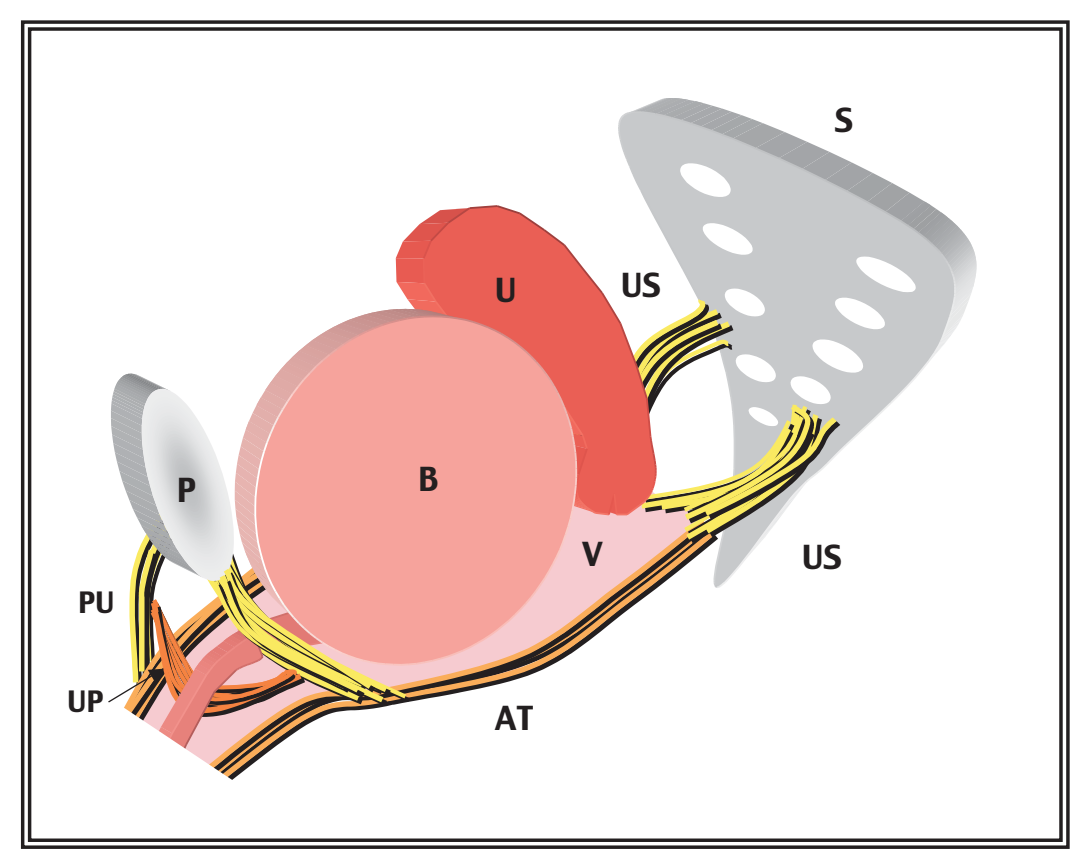

FIGURA 4. Representación esquemática de los principales ligamentos de sustentación de la pared vaginal anterior. P: pubis; U: útero; V: vagina; S: sacro; AT: arco tendineo; PU: ligamento pubouretral; UP: ligamento uretropélvico; US: ligamento útero sacro. 
vesical es denominada la zona de elasticidad crítica por su comportamiento dinámico y considerado fundamental en el mecanismo de micción y de continencia urinaria (Fig. 5).

Papel de tensión vaginal en la micción y la continencia

Durante el reposo en la mujer normal, la pared vaginal anterior y la fascia pubocervical se encuentran suspendidas en forma anterior por los ligamentos pubouretrales, lateralmente por el arco tendíneo de la fascia pélvica y en forma posterior por los ligamentos útero sacros. En esta situación existe tensión de la pared vaginal en tres direcciones: (a) anteriormente, por la contracción del eje pubococcígeo del elevador del ano; (b) posteriormente por el plató del elevador del ano (constituido por la porción posterior del músculo pubococcígeo y por los músculos ílio e

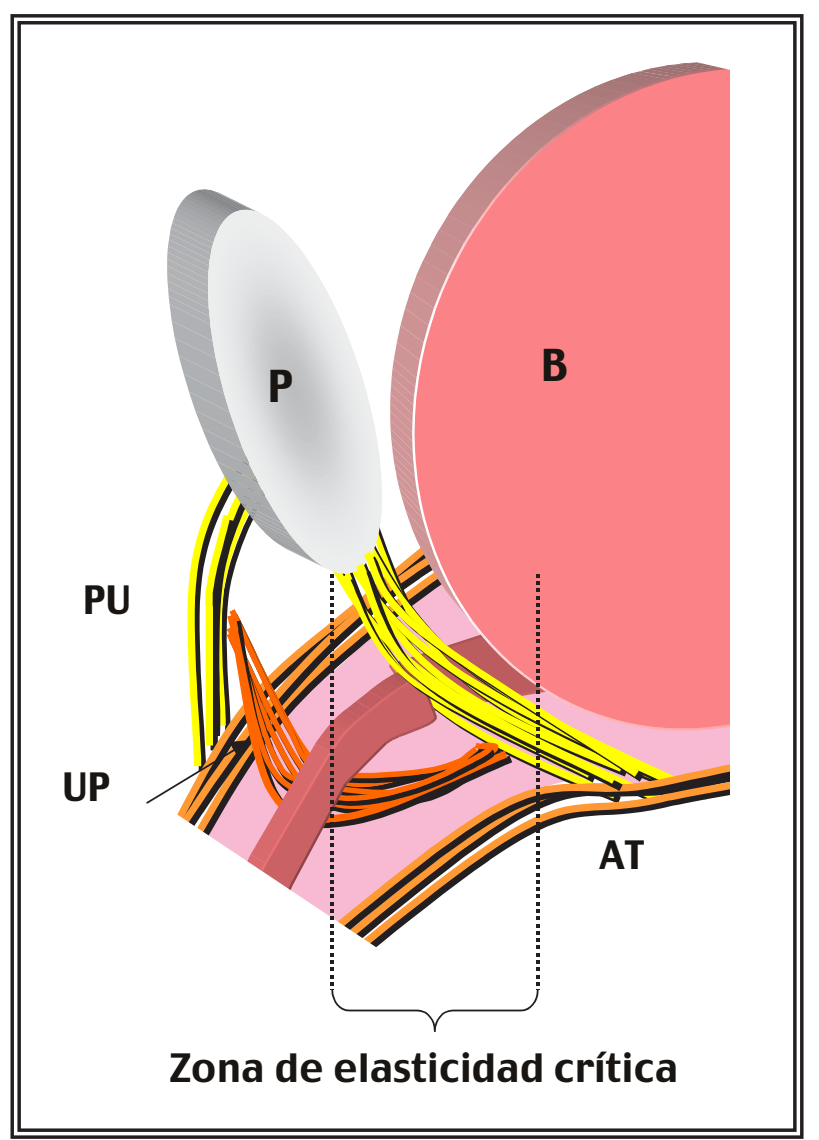

FIGURA 5. La zona de elasticidad crítica está comprendida entre el tercio uretral medio y el cuello vesical. P: pubis; B: vejiga; UP: ligamento uretropélvico; PU: ligamento pubouretral; AT: arco tendineo de la fascia pélvica. isquiococcígeos) e (c) inferiormente, por el músculo longitudinal del ano (que se extiende desde la fascia del músculo elevador del ano hasta la piel de la región perianal, compuesta por fibras de los músculos pubococcígeo, pubouretral e ileococcígeo) (Fig. 6). La contracción de eje pubococcígeo contra la cara posterior del pubis determina la tracción superior y anterior de los ligamentos pubouretrales y uretropélvicos, resultando en el cierre e inmovilización del tercio medio de la uretra. Simultáneamente, en la situación de reposo, la vejiga es traccionada posteriormente hacia abajo por la contracción del plató del elevador y por el músculo longitudinal del ano, determinando el cierre del cuello vesical. La integridad de los ligamentos cardinales, útero sacros y del septo recto vaginal son fundamentales para las transmisiones nerviosas locales, responsables del reflejo miccional.

Durante el reposo, la musculatura estriada periuretral, también denominada rabdoesfinter, determina la tensión adicional sobre la musculatura uretral, creando un efecto de "sello mucoso" que es mas eficiente en la medida en que es mejor el tropismo mucoso y está mejor desarrollado el plexo vascular submucoso. Estudios electromiográficos demostraron que el rabdoesfinter presenta dos tipos de fibras, siendo un grupo de contracción lenta y tónica y otro de fibras de contracción rápida, que son activadas por reflejo cuando ocurre aumento súbito de la presión abdominal.

Durante la micción, de forma inversa, ocurre el relajamiento del eje pubococcígeo, causando disminución de la tensión aplicada por los ligamentos pubouretrales sobre la vagina y consecuentemente sobre la uretra. Esto permite que la tracción en dirección posterior aplicada por el plató del elevador y por el músculo longitudinal del ano sobre la fascia pubocervical determine la abertura y embudización del cuello vesical (Fig. 7). Simultáneamente, ese estiramiento causaría la activación de las terminaciones nerviosas responsables por el desencadenamiento del reflejo miccional. Este seria estimulado, también, por el contacto de la orina con la uretra proximal, igualmente se ha descrito la presencia del cuello vesical abierto durante el reposo, en mujeres continentes. 


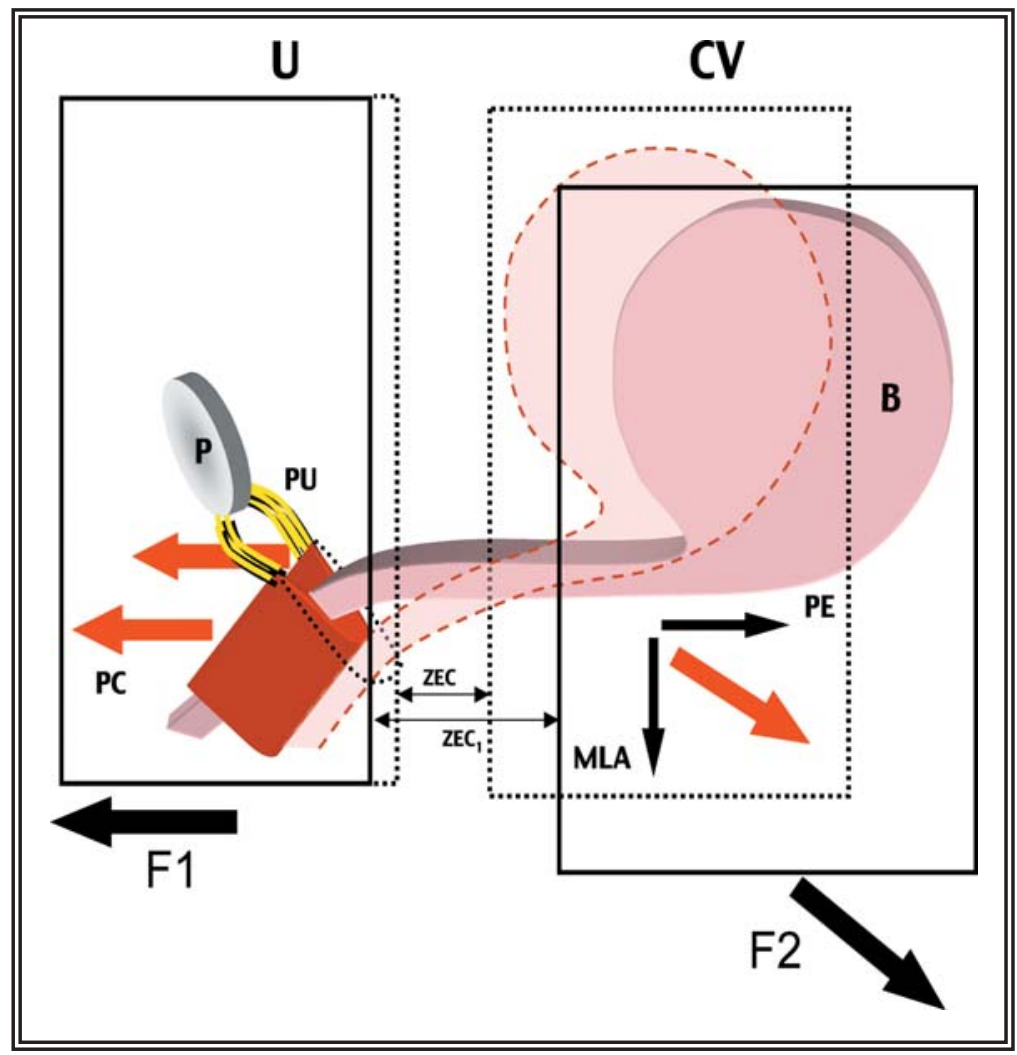

FIGURA 6. Mecanismo de apertura y cierre de la uretra y del cuello vesical. U: mecanismo de cierre uretral; CV: mecanismo de cierre del cuello vesical; ZEC: zona de elasticidad critica; ZEC1: zona de elasticidad critica durante la micción o el esfuerzo abdominal; F1: fuerza aplicada en dirección anterior; PC: músculo pubococcígeo; F2: fuerza resultante aplicada en dirección posterior $e$ inferior; PE: plató elevador; MLA: músculo longitudinal del ano. Los mecanismos de cierre uretral $(\mathrm{U})$ y del cuello vesical $(\mathrm{CV})$ son independientes y exigen una elasticidad adecuada de los tejidos locales para su eficiencia. Desde el punto de vista funcional, tal elasticidad es imprescindible en la zona de elasticidad critica. Las dos fuerzas resultantes en dirección opuesta que actúan sobre la vagina (F1 y F2). F1 es el resultado de la contracción del eje pubococcígeo del elevador del ano y determina un aumento de tensión sobre la uretra, generando su cierre. F2 es la resultante posteroinferior de la contracción del plató elevador y del músculo longitudinal del ano y puede determinar el cierre del cuello vesical en reposo y su apertura cuando la resultante F1 disminuye, por relajación del músculo pubococcigeo. La pérdida de la elasticidad de la ZEC, puede determinar la apertura del cuello vesical igualmente cuando hay intención de su cierre, debido a que F2 se torna mayor que F1.

\section{CLASIFICACIÓN DE LOS DEFECTOS}

Dependiendo de la localización de la lesión músculo fascial o ligamentar y de la sensibilidad de las terminaciones nerviosas locales, se puede desarrollar incontinencia de esfuerzo, incontinencia de urgencia, alteraciones del vaciamiento vesical o varias combinaciones de estas condiciones. De manera general, existen 6 defectos básicos que deben ser investigados sistemáticamente (Fig. 8):

1) Defecto del soporte suburetral ("Hammock")

2) Sindrome de vagina fija ("Tethered vagina syndrome")

3) Distensión de los ligamentos pubouretrales

4) Distensión de los ligamentos útero sacros y del soporte del ápice vaginal

5) Lesiones de la inserción vaginal de los músculos pubococcígeos

6) Lesiones de los músculos estriados del piso pélvico

a) Traumatismo del esfinter externo del ano

b) Distensión, parálisis o ruptura de las inserciones del plató elevador
Otra forma de clasificación anatómica, menos específica pero igualmente útil, corresponde a la definición de tres zonas de disfunción (anterior, média y posterior), de acuerdo con la localización predominante de los defectos (Fig. 9).

De forma general, las disfunciones de la zona anterior determinan incontinencia urinaria de esfuerzo, mientras los defectos posteriores causan, más frecuentemente, alteraciones de vaciamiento vesical. Síntomas como polaquiuria, urgencia y nocturia pueden ocurrir en ambas situaciones. La presencia de un defecto puede representar un significado clínico, debido a los mecanismos compensatorios específicos que pueden estar presentes en una paciente determinada.

\section{Defecto del soporte suburetral ("Hammock")}

En este defecto de observa la distensión de la porción suburetral de la pared vaginal. Puede ser investigado a través del pinzamiento de la pared vaginal, dando como resultado la disminución o la desaparición de la pérdida urinaria durante el esfuerzo abdominal solicitado (Fig. 10). Esta alteración se asocia, frecuentemente a la distensión 

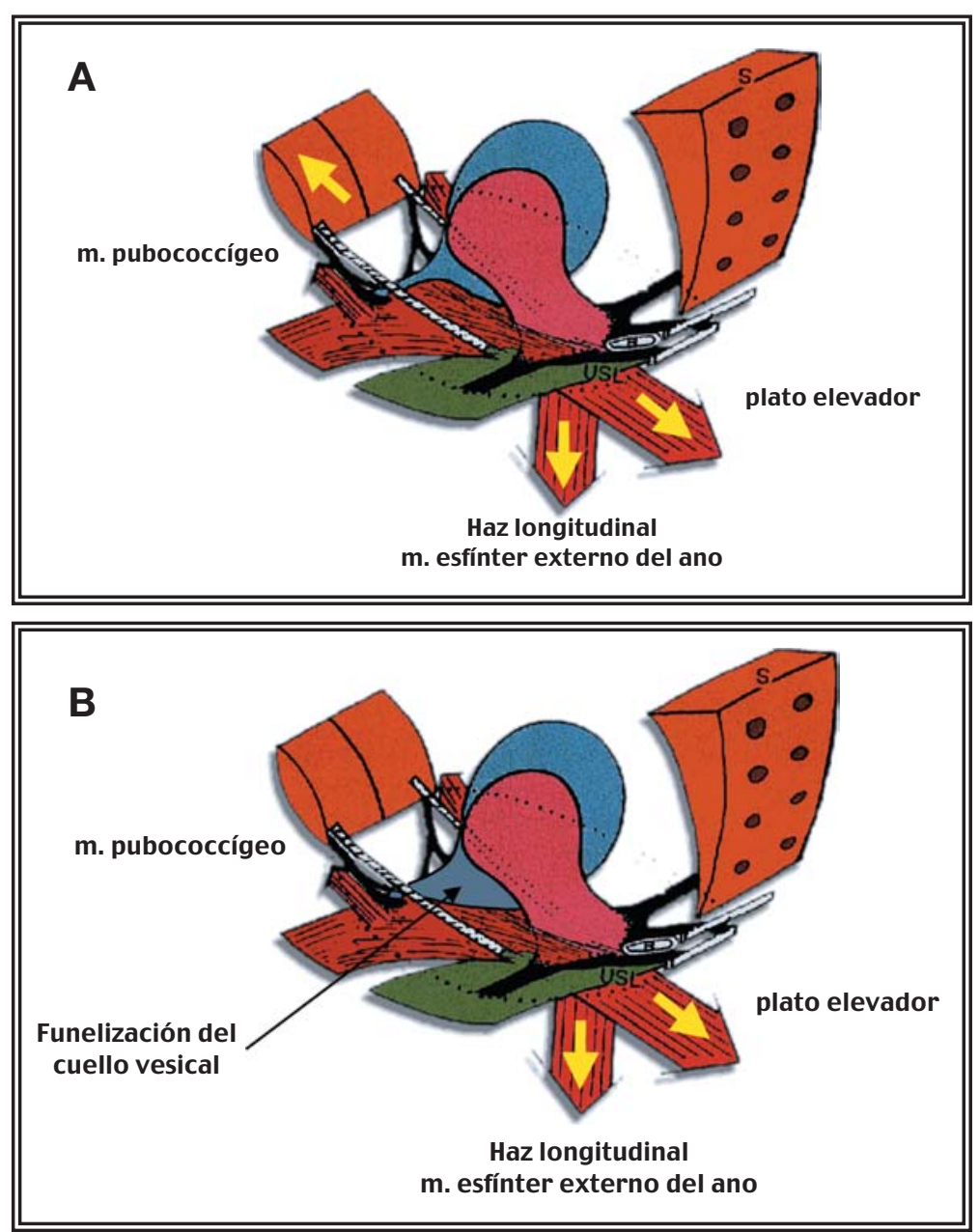

FIGURA 7. (A) En reposo, hay un equilibrio entre la tensión aplicada en dirección anterior y por el eje pubococcígeo del elevador del ano y posteroinferior por el plató del elevador del ano y por el músculo longitudinal del ano.

(B) Durante la micción, existe predominio de la tensión aplicada en dirección posterior por el plató del elevador del ano y por el músculo longitudinal del ano en relación a la ejercida por el eje pubococcígeo del elevador del ano. Esto determina la apertura y alteración del cuello vesical y la disminución de la tensión de los ligamentos sobre el tercio uretral medio. de los ligamentos pubouretrales o a la avulsión de su inserción vaginal, que determina un desajuste del equilibrio entre la tensión aplicada anterior y posteriormente, con predominio de la tensión aplicada en dirección posterior por el plató del elevador y por el músculo longitudinal del ano en relación a la tensión anterior del músculo pubococcígeo. Habitualmente, en esta situación, la pérdida urinaria ocurre a los mismos esfuerzos y podrá ser por este motivo mayor o menor dependiendo del "sello mucoso" que se relaciona directamente con las condiciones tróficas de la vagina. Frecuentemente, se verifica aumento de la pérdida después de la inserción de un espéculo apoyado sobre la pared vaginal posterior con discreta tracción en dirección al fórnix posterior de la vagina, debido a que un estiramiento vaginal posterior determina la disminución adicional del soporte suburetral en esa situación.

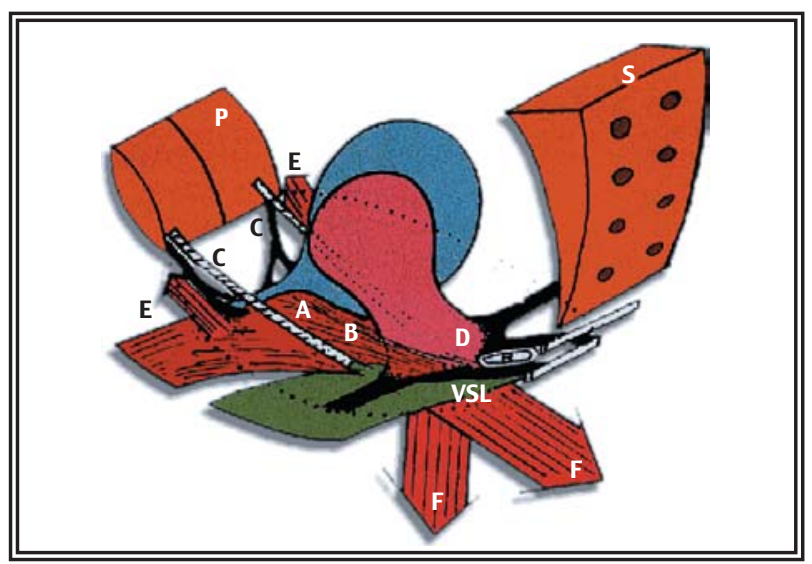

FIGURA 8. Localización de los defectos más frecuentemente encontrados en el suelo pélvico femenino. (A) Defecto de soporte suburetral ("Hammock"); (B) retracción cicatrizal de la vagina ("Tethered vagina sindrome"); (C) Distensión de los ligamentos pubouretrales; (D) Distensión de los ligamentos útero sacros y del soporte del ápice vaginal; $(E)$ Lesiones de la inserción vaginal de los músculos pubococcigeos; (F) Lesiones de los músculos estriados del piso pélvico. USL: Ligamento útero sacro; S: sacro; P: pubis. 


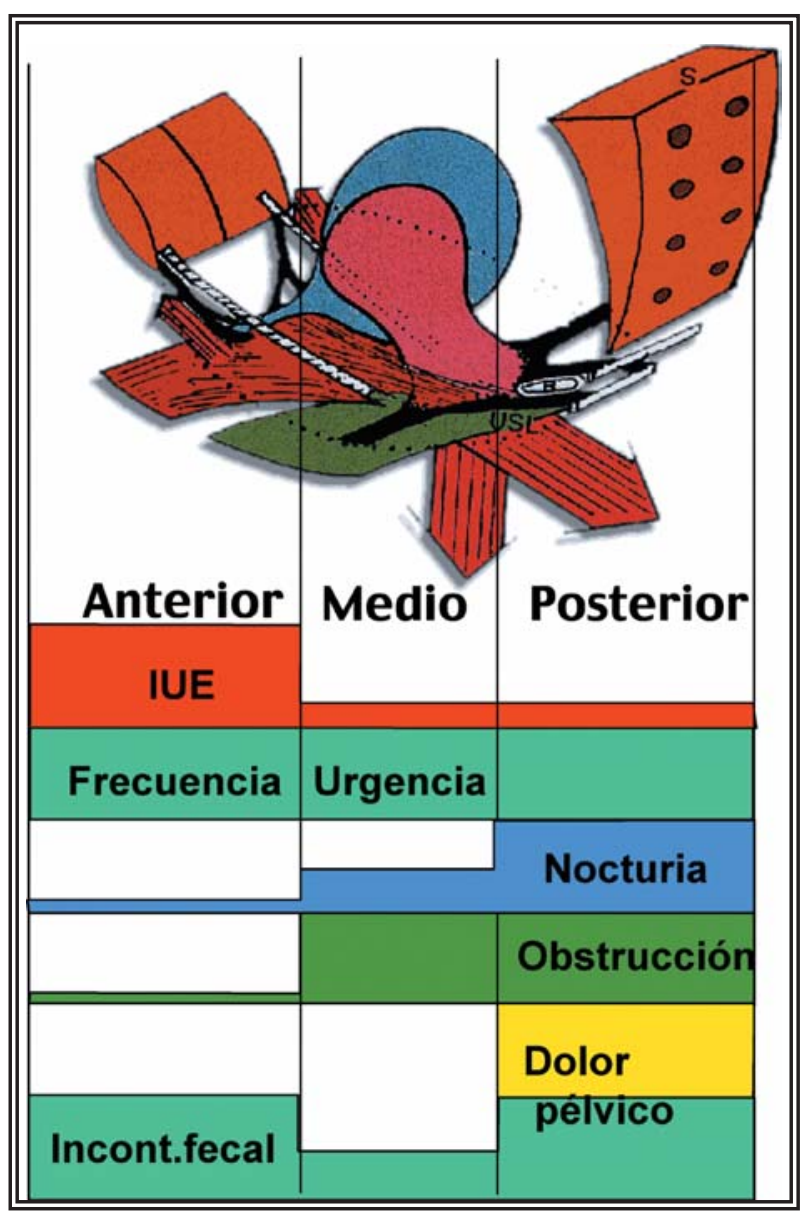

FIGURA 9. Origen de los sintomas más frecuentes rela cionados con el piso pélvico femenino, de acuerdo con la teoria integral. Se observan tres zonas de disfunción: anterior, media $y$ posterior.

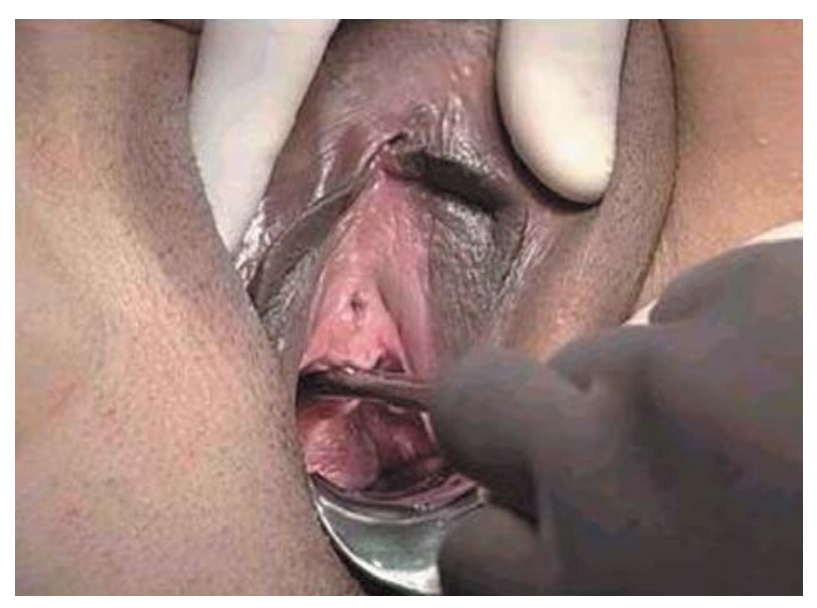

FIGURA 10. En el defecto del soporte suburetral ("Hammock") se observa la disminución o desaparición de la pérdida urinaria con el pinzamiento de la pared vaginal.
Distensión de los ligamentos pubouretrales

Puede ser congénita, ocurrir con el envejecimiento (alteraciones colágenas) o secundarias a las lesiones ocurridas durante el parto vaginal. Habitualmente las pacientes presentan incontinencia urinaria asociada a constipación intestinal. Puede ser evaluada aplicándose una pinza lateralmente a la uretra, a nivel del tercio medio y solicitando a la paciente que realice maniobras de esfuerzo abdominal (Fig. 11). En esta prueba, derivado del clásico test de Bonney, se evalúa la inserción vaginal de los ligamentos pubouretrales aisladamente. La disminución de la pérdida urinaria después de la aplicación de la pinza será mayor en la medida en que el defecto en el ligamento respectivo sea mayor. En general ese defecto ocurre en forma concomitante con la pérdida del soporte suburetral y su corrección se realiza conjuntamente

Retracción cicatricial de la vagina ("Tethered vagina syndrome")

Cirugias previas del tipo colporrafia anterior o para la elevación del cuello vesical pueden comprometer la elasticidad de la pared vaginal anterior, fijando el músculo pubococcígeo al plató del elevador. En esta situación el equilibrio dinámico entre los vectores de la tensión anterior y posterior está comprometido y la incontinencia es acentuada. $\mathrm{Al}$ examen físico, se observa la retracción cicatrizal no elástica al nivel del cuello vesical, con disminución del acomodamiento de la pared vaginal anterior. Habitualmente, la paciente no consigue interrumpir la pérdida, que ocurre generalmente incluso en reposo. El tratamiento implica la restauración de la elasticidad de la pared vaginal anterior, sea a través de injertos de pared vaginal posterior (caso excedente), cutáneos o segmentos en forma de zetaplastia (Fig. 12).

Distensión de los ligamentos útero sacros y del soporte del ápice vaginal

Cuando este defecto está presente, la paciente puede referir incontinencia urinaria, dolor pélvico y eventualmente, podrá presentar alteraciones de la fase miccional y residuo postmiccional elevado. La distensión del fórnix posterior durante el parto y la sutura de la cúpula vaginal durante la histerectomía sin atención de sus inserciones ligamentosas, son los dos factores etiológicos más 

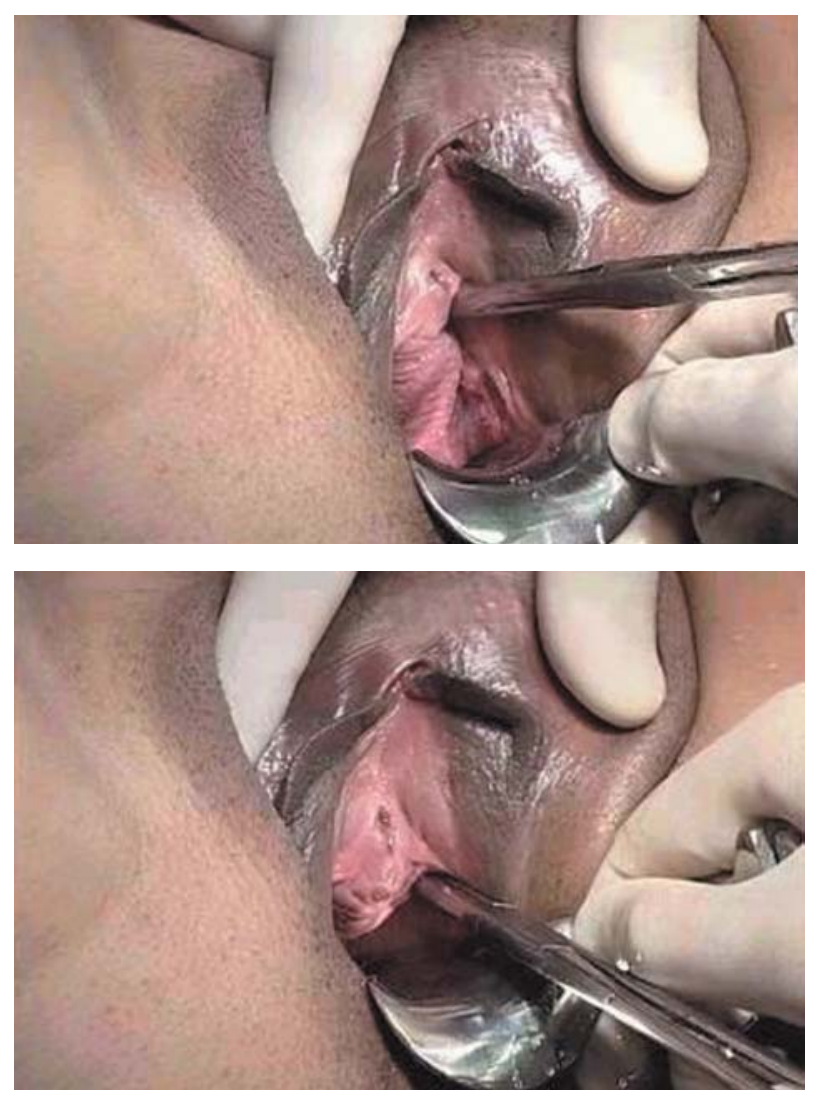

FIGURA 11. Demostración de la distensión de los ligamentos pubouretrales. (A) La disminución de la pérdida urinaria después de la aplicación de la pinza será mayor cuanto mas importante fuese el defecto respectivo en el ligamento. (B) Después de la disminución de la tensión local, la pérdida urinaria debe volver a ser observada.

importantes, sin embargo en forma menos frecuente, pueden ocurrir malformaciones congénitas que se tornan sintomáticas después del nacimiento. El resultado de la tensión inadecuada de la fascia pubocervical y de la vagina puede determinar síntomas como frecuencia, urgencia y nocturna. $\mathrm{Al}$ examen se observa disminución del compartimiento vaginal, con abombamiento de la cúpula, generalmente debido a un enterocele o a un rectocele proximal asociado. Con una paciente con vejiga llena, es posible probar la desaparición de los síntomas con el pinzamiento seguido de la elevación del fórnix posterior, solicitándole que realice maniobras de valsalva conjuntamente (Fig. 13).

Lesiones de la inserción vaginal de los músculos pubococcígeos

Resulta en sintomas semejantes a los de la distensión de los ligamentos pubouretrales. La vagina en forma natural está adherida a la cara inferior del músculo pubococcígeo por un tejido conjuntivo denso. La tensión exagerada en este punto puede alterar el mecanismo de cierre del cuello vesical y la transmisión de la presión ejercida sobre los ligamentos pubouretrales, comprometiendo el cierre uretral. Este defecto puede corresponder a la lesión descrita por Bailey como tipo 2B o defecto del soporte inferior de la vagina ${ }^{1}$ o al defecto paravaginal referido por Richardson ${ }^{2,3}$.
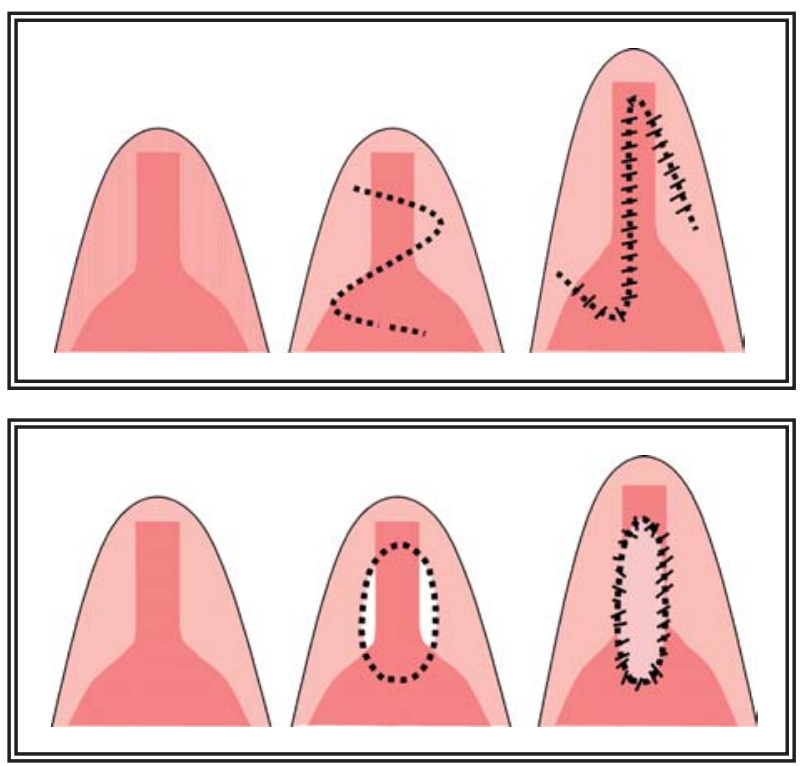

FIGURA 12. Corrección quirúrgica de la retracción cicatrizal de la vagina ("tethered vagina syndrome"). (A) zeta plastia; (B) injerto de pared vaginal posterior o de la piel.

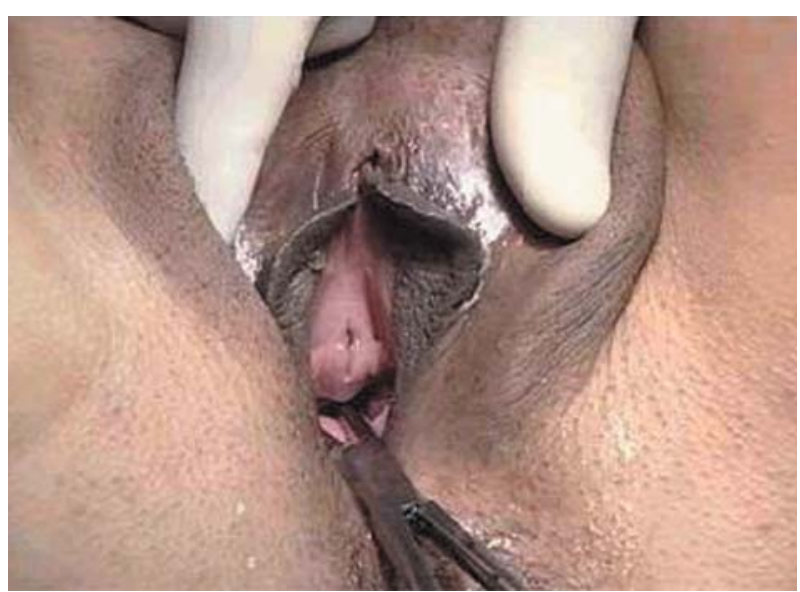

FIGURA 13. Prueba de la distensión de los elementos de soporte del ápice vaginal. Habitualmente esa maniobra determina la disminución de la pérdida urinaria cuando es realizada conjuntamente con la maniobra de valsalva. La paciente puede referir, además, disminución del deseo miccional y del dolor pélvico, caso presente. 
Clínicamente la situación es semejante a cuando se da la distensión de los ligamentos pubouretrales, pero la diferenciación tiene un significado académico, ya que ambos defectos son habitualmente corregidos en forma simultánea con la mayoría de las técnicas del Sling.

Lesiones de los músculos estriados del piso pélvico

No todas las pacientes con parálisis de los músculos del piso pélvico presentan incontinencia urinaria y/o fecal. La parálisis muscular puede ser explicada como consecuencia de la lesión de las terminaciones motoras ocurrida durante un expulsivo prolongado, lo cual puede determinar, no obstante, las lesiones del tejido conjuntivo de la vagina ${ }^{4}$. La parálisis puede determinar alteraciones de la posición de las vísceras pélvicas en reposo e inducir a una deficiencia mecánica debido a alteraciones de los ángulos de las fuerzas aplicadas en el ligamento pubouretral. La incontinencia urinaria puede, por lo tanto, derivarse de la lesión directa o por la abulsión del músculo pubococcígeo, que es una causa obstétrica muy rara en la actualidad.

\section{La Teoria Integral en la Cirugía Reconstruc- tiva Pélvica}

Alteraciones tisulares y éxito quirúrgico

La vagina y sus ligamentos de soporte presentan tres funciones básicas en la estática de los órganos pélvicos femeninos:

a) Arquitectura elástico para los mecanismos de cierre uretral y del cuello vesical.

b) Órgano transmisor de las fuerzas generadas por la contracción de los músculos del piso pélvico.

c) Soporte de las terminaciones nerviosas de la base vesical, previniendo su activación prematura.

Como cualquier otro órgano, la vagina se encuentra sujeta a alteraciones del envejecimiento, que determina pérdida de la elasticidad y de la vitalidad. Puede además sufrir lesiones ocurridas durante el período expulsivo del parto, resultando en herniaciones. En este contexto, las razones para un in suceso quirúrgico del tratamiento de la incontinencia urinaria y de los prolapsos urogenitales pueden suceder por varios factores: (a) alteraciones vaginales relacionadas a hipoestro- genismo; (b) distensión de la porción suburetral y del fórnix posterior; (c) alteraciones fibróticas resultantes de procedimientos repetidos; (d) Compromiso de la adherencia natural colágena, entre la vagina y el músculo pubocccígeo.

La resistencia de los tejidos de la vagina disminuye naturalmente durante el envejecimiento, las episiotomías y el estiramiento quirúrgico contribuyen aún mas para su deterioro funcional. De esta forma se recomienda evitar cualquier resección innecesaria del tejido vaginal. Segmentos vaginales desepitelizados (o en los cuales el epitelio fue electrocauterizado) pueden, incluso, ser útiles en el refuerzo de la fascia perineal, durante las colporrafias posteriores ("Bridge Technique") en el reparo de la fascia pubocervical, anotando la importancia de la preservación del tejido vaginal.

La elasticidad vaginal acumula energía potencial resultante de las tensiones aplicadas sobre ella por los ligamentos y músculos del piso pélvico. Tal energía tendrá que ser redistribuida en el caso que una porción de la vagina sea removida, aumentando el riesgo de las dehiscencias en el período postoperatorio inmediato y de disfunciones del piso pélvico en forma tardía, especialmente cuando sea sometida a esfuerzo, como toser o durante el coito.

Igualmente, incluso cuando la tensión vaginal se observa adecuada, durante el procedimiento quirúrgico puede haber deterioro postoperatorio, resultante de las alteraciones visco elásticas del tejido conectivo vaginal.

\section{El papel del cirujano}

Un diagnóstico incorrecto del defecto anatómico compromete el resultado quirúrgico y puede determinar nuevas disfunciones del suelo pélvico. Es común el relato del desarrollo de enteroceles después de cirugías antiincontinencia, debido a la falta de diagnóstico del defecto asociado en el fórnix posterior.

Síntomas como urgencia miccional y dolor pélvico pueden ser determinados inicialmente por defectos del fórnix posterior y pueden ser investigados durante el examen físico preoperatorio, traccionando el fórnix posterior con una pinza o igualmente con una de las valvas del espéculo vaginal, estando el paciente con la 
vejiga llena. En los casos donde hubiere defecto del soporte del fórnix posterior, resultante de la lesión de la inserción vaginal de los ligamentos útero sacros, esa maniobra produce la desaparición de la urgencia y la disminución de la sensación de dolor. Las lesiones del fórnix posterior ocurren generalmente por no incluir el extremo del complejo cardinal-útero sacro durante la sutura de la cúpula vaginal en las histerectomías.

Otro problema común en las cirugías antiincontinencia, se relaciona con la retracción cicatrizal de la vagina ("Tethered vagina syndrome"). Tal alteración puede inmovilizar la uretra, dificultando el proceso de cierre al comprometer la zona de elasticidad crítica. El estiramiento de la vagina altera su elasticidad y la capacidad de acumular energía potencial. Las cirugías vaginales y antiincontinencia deben siempre considerar los efectos de la cicatrización y del proceso de envejecimiento sobre los tejidos vaginales, que llevan a un proceso de estrechamiento natural. La recidiva tardía de la incontinencia se relaciona con las alteraciones titulares de la vagina, particularmente de su matriz conjuntiva, así como las fibras colágenas y elásticas. La disección de las adherencias entre la vagina y la uretra al igual que la sutura vaginal mediante la técnica de zeta plastias o con injertos, pueden en forma aislada solucionar la incontinencia, en el caso que el soporte uretral sea adecuado, conferido por los ligamentos pubouretrales que estuviesen preservados.

El principal paradigma de la corrección del defecto del soporte suburetral ("Hammock") es la conservación del eje vaginal, sin elevaciones o angulaciones de la uretra. Teóricamente ese defecto podría ser corregido por la plicatura exclusiva del tejido suburetral. Mientras tanto, la plicatura exclusiva de la fascia pubocervical, que es la técnica clásica de colporrafia anterior resulta en un índice de éxito inferior al 50\% en el seguimiento prolongado. Como el soporte suburetral depende de la acción conjunta de los ligamentos pubouretrales y del tejido suburetral, se considera que la corrección debe ser conjunta.

La distensión de los ligamentos pubouretrales y las lesiones de la inserción vaginal de los músculos pubococcígeos son responsables por las alteraciones más significativas del mecanismo de cierre uretral. Cirugías que inmovilizan el cuello vesical, como las colposuspensiones retropúbicas y los Slings aplicados sobre el cuello vesical, pueden comprometer la zona de elasticidad crítica y dificultan la tracción craneal de la uretra ejercida por los ligamentos pubouretrales. Tales técnicas se relacionan con un elevado riesgo potencial de disfunciones miccionales y obstrucción infravesical postoperatoria.

El proceso de la abertura uretral exige que el ápice vaginal esté adecuadamente fijado por el complejo cardinal y útero sacro. La distensión de los ligamentos útero sacros y del soporte del ápice vaginal se manifiesta clínicamente a través del desarrollo de rectoceles proximales y de enteroceles, los cuales deben ser corregidos conjuntamente con los procedimientos anti incontinencia, con el riesgo de retención urinaria post operatoria. En las histerectomías abdominales, la sutura transversal de la cúpula vaginal puede generar un vaciamiento vesical incompleto. La sutura longitudinal en "bolsa" de la cúpula vaginal promueve un mejor soporte, previniendo las disfunciones, debido a que promueven la tensión central de los ligamentos útero sacros.

\section{Agradecimiento}

Agradecemos al Prof. Peter Petros por los conceptos, entrenamientos y parte del material didáctico.

\section{REFERENCIAS}

1. Bailey KV. Clinical investigation into uterine prolapse with stress incontinence: treatment by modified Manchester colporaphy. Journal of Obstetrics \& Gynaecology of the British Emp. Part II, 1956;63:663-676.

2. Richardson AC, Edmonds PB, Williams NL. Treatment of stress incontinence due to a paravaginal fascial defect. Obst. Gynecol. 1980;57,3:357-362.

3. Baden WF, Walker TA. Urinary stress incontinence: Evolution of paravaginal repair. The Female Patient 1987;89-105.

4. Petros PE, Ulmsten U. An Integral Theory of Female Urinary Incontinence. Acta Scand O\&G. 1990, Supplementum 153, 69:1-79.

Dr. C. Ricceto

Universudade Estadual de Campinas

UNICAMP, Campinas. Sao Paulo. Brasil

(Trabajo recibido el 8 septiembre 2004) 